\title{
Signet-Ring Cell Carcinoma of the Colon: A Case Report and Review of the Literature
}

\author{
Peter Y. Park ${ }^{a}$ Teresa Goldin ${ }^{b} \quad$ John Chang ${ }^{c}$ Maurie Markman ${ }^{d}$ \\ Madappa N. Kundranda ${ }^{c}$ \\ ${ }^{a}$ A.T. Still University, Kirksville College of Osteopathic Medicine, Kirksville, Mo., \\ ${ }^{b}$ Western Regional Medical Center at Cancer Treatment Centers of America, \\ Goodyear, Ariz., 'Banner MD Anderson Cancer Center, Gilbert, Ariz., and \\ ${ }^{\mathrm{d}}$ Cancer Treatment Centers of America, Medicine and Science, Philadelphia, Pa., USA
}

\section{Key Words}

Colorectal adenocarcinoma - Signet-ring cell carcinoma - Mucinous adenocarcinoma .

Adjuvant chemotherapy · Microsatellite instability

\begin{abstract}
Background: Colorectal adenocarcinoma (CRC) is the third leading cause of death in the United States. One of the histologic subtypes of CRC is signet-ring cell carcinoma (SRCC), which has a distinct molecular and tumor biology from that of adenocarcinoma. Primary SRCC diagnosed at an early stage is very rare as most cases are detected at an advanced stage. Therefore, overall prognosis of SRCC is poor. Case Presentation: A 36-year-old female presented to her primary care physician with new-onset progressive right lower quadrant pain without any significant past medical or family history. Computed tomography scan of the abdomen and pelvis with contrast showed a $4.9 \times 3.5 \times 3.1 \mathrm{~cm}$, lobulated, septated cystic mass arising from the cecum. The mass demonstrated wall enhancement and contained focal areas of coarse calcification. There was nodal involvement either locally or distally. The patient underwent right hemicolectomy, and pathology revealed a high-grade mucinous carcinoma with signet-ring cell variant invading through the muscularis propria and into the subserosal adipose tissue. The margins were negative for tumor, and no lymphovascular or perineural invasion was noted. None of the 14 resected pericolonic lymph nodes was positive for malignancy. Hence, she was staged as pT3, pNO, pMx-stage IIA. The appendix was not involved. Microsatellite instability testing showed the preservation of MLH1, PMS2, MSH2 and MSH6 proteins by IHC and PCR. Carcinoembryonic antigen level was within normal limits. Due to the patient's young age, aggressive histology and microsatellite-stable status, adju-
\end{abstract}


Park et al.: Signet-Ring Cell Carcinoma of the Colon: A Case Report and Review of the Literature

vant fluropyrimidine (5-FU)-based therapy with the single agent capecitabine was initiated. The patient completed 6 months of adjuvant therapy and has been disease free for approximately 18 months. Conclusion: Primary SRCC of the cecum is a rare disease. Given the poor prognosis of these patients, early-stage disease with microsatellite-stable patients should be considered for adjuvant 5-FU-based therapy in an attempt to prevent recurrence.

(C) 2015 The Author(s)

Published by S. Karger AG, Basel

\section{Introduction}

Colorectal adenocarcinoma (CRC) is one of the most common cancers worldwide and the third leading cause of death in the United States [1]. CRC can be histologically subtyped into adenocarcinoma (AC), which accounts for the large majority of cases; mucinous AC; signet-ring cell carcinoma (SRCC), and other even less frequent subtypes [2,3]. Primary SRCC of the colon is a rare entity, accounting for less than 1\% of all CRC [4]. SRCC is associated with a higher-grade tumor associated with young age, female patients, and distinct molecular patterns, such as microsatellite instability (MSI) and activating mutations of the BRAF gene compared to mucinous AC $[3,5]$. However, since clinical symptoms tend to occur late in the course of SRCC, most cases are usually detected at an advanced stage with a poor overall survival rate [3, 5-7]. Primary SRCC at an early stage is rare, and only 27 cases have been reported [8].

We report a case of a 36-year-old female who presented with progressive right lower quadrant pain and was diagnosed with primary SRCC of the cecum. Her surgical resection showed stage IIA without evidence of metastasis or nodal involvement. The patient responded well without any adverse reactions to an adjuvant fluropyrimidine (5-FU)-based therapy with oral capecitabine.

\section{Case Report}

A 36-year-old female presented to her primary care physician with progressive right lower quadrant abdominal pain without any significant past medical and family history. Computed tomography (CT) scan of the abdomen and pelvis with contrast showed a $4.9 \times$ $3.5 \times 3.1 \mathrm{~cm}$, lobulated, septated cystic mass arising from the cecum. The mass demonstrated wall enhancement with multiple coarse calcifications and ascites inferior to the mass (fig. 1). No lymphadenopathy was seen on CT scan. She subsequently underwent surgical resection.

The specimen revealed organ-confined disease with final pathological staging IIA (pT3, pN0, pMx). High-grade mucinous carcinoma with signet-ring cells invaded the muscularis propria into the subserosal adipose tissue (fig. 2). The pathological specimen had negative margins and showed no lymphovascular or perineural invasion. Fourteen resected lymph nodes revealed no nodal metastasis $(0 / 14)$. The cells clustered into various sizes of clusters and gland-like structures at $\times 40$ magnification. At $\times 400$ magnification, the moderately differentiated malignant cells formed classical columnar strips (fig. 3). At $\times 600$ magnification, the cells floated within the abundant extracellular mucin pools as either clusters or isolated single cells (fig. 4). Molecular study specific for MSI showed the preserved protein expression of MLH1, PMS2, MSH2 and MSH6. MSI by PCR was noted to be stable. Carcinoembryonic antigen was within the normal limit $(<0.3 \mathrm{ng} / \mathrm{ml})$. 
Park et al.: Signet-Ring Cell Carcinoma of the Colon: A Case Report and Review of the Literature

Due to the patient's young age, early-stage disease, and aggressive high-grade mucinous carcinoma with microsatellite stability, an adjuvant 5-FU-based therapy with oral capecitabine was commenced to prevent recurrent disease. The patient has completed 4 of the planned 8 cycles and has tolerated and responded to the therapy without any significant hematological or nonhematological toxicities.

\section{Discussion}

Based on several reports including a large population-based study of 197,757 CRC patients, SRCC is a distinct entity based both on clinical presentation and pathology [9]. SRCC occurs in the younger population with female predominance, usually less than 40 years of age $[5,10,11]$. More than $96 \%$ of cases of SRCC arise in the stomach with the rest occurring in the colon, rectum, gallbladder, pancreas, urinary bladder, and breast $[10,12]$. SRCC usually presents at an advanced stage with node-positive disease and metastatic spread, which results in poor prognosis $[3,5,13]$. A unique feature of SRCC is its fibrotic appearance and preference for peritoneal metastasis over the liver $[10,14]$.

Pathologically, SRCC demonstrates unique features that distinguish the cells from routine $\mathrm{AC}$ of the colon and confer aggressiveness to these cells. The signet-ring, malignant cells are seen floating in abundant extracellular mucin pools either as clusters or isolated cells. These cells have a slightly lower prevalence of KRAS mutation but a higher BRAF mutation rate as compared to classical AC [5, 15], and the prognostic implication is unknown. Loss of E-cadherin expression has been reported and contributes to the high-grade and invasive nature of SRCC as the cells acquire stem cell-like characteristics $[5,16]$. MSI tends to be more prevalent in SRCC than AC [5]. There is some evidence that SRCC patients with stage II disease do not benefit from adjuvant therapy, whereas stage III patients with high MSI benefited from single-agent 5-FU adjuvant therapy [17].

The overall prognosis of SRCC in general is similar to other cancers in that staging and grade are important determinants. The available literature suggests that the tumor staging is the best predictive factor for the prognosis of SRCC of the colon where higher tumor staging means poorer prognosis $[5,6]$. The 5-year survival rate in SRCC of the colon ranges from 0 to $12 \%$, and disease recurrence is more frequent in SRCC of the colon compared to AC [18]. Absence of lymphovascular invasion, lack of lymph node metastasis, and lower TNM stage had a favorable effect on the survival of SRCC patients [13]. Patients with SRCC can definitely benefit from closer follow-up or even intensified adjuvant therapy due to their high rates of local and distant recurrence [3, 9]. In a study by Hugen et al. [9], adjuvant chemotherapy for SRCC stage III of the colon was associated with improved survival.

Our patient is atypical compared to the cases generally reported in the literature. Other than presenting with abdominal pain and being microsatellite stable, her SRCC occurred in the cecum and had invaded the subserosal fat. Instead of having a fibrotic appearance, her cancer is more cystic in appearance than the generally reported SRCC. Due to early disease of stage IIA and microsatellite stability in our patient, an adjuvant 5-FU-based therapy with oral capecitabine was initiated to prevent recurrence of SRCC. At the time of publication, the patient remained disease free. 
Park et al.: Signet-Ring Cell Carcinoma of the Colon: A Case Report and Review of the Literature

\section{Conclusion}

Primary SRCC is considered a distinct entity based on the clinical and pathological features. In this case report, we have shown a rare case of primary SRCC of the cecum. Given the poor prognosis of these patients, early disease of stage II in microsatellite-stable patients should be considered for adjuvant 5-FU-based therapy in an attempt to prevent recurrence.

\section{Statement of Ethics}

The authors have no ethical conflicts to disclose.

\section{Disclosure Statement}

The authors declare that there are no conflicts of interest.

\section{References}

Siegel R, Ma J, Zou Z, Jemal A: Cancer statistics, 2014. CA Cancer J Clin 2014;64:9-29.

2 Bosman FT, Carneiro F, Hruban RH, et al: WHO classification of tumours of the digestive system, ed 4. Geneva, World Health Organization, International Agency for Research on Cancer, 2010.

-3 Ulrich N, Zimmermann A, Spath C, et al: Mucinous and signet-ring cell colorectal cancers differ from classical adenocarcinomas in tumor biology and prognosis. Ann Surg 2013;258:775-783.

4 Verhulst J, Ferdinade L, Demetter P, et al: Mucinous subtype as prognostic factor in colorectal cancer: a systemic review and meta-analysis. J Clin Pathol 2012;65:381-388.

5 Gopalan V, Smith RA, Ho YH, et al: Signet-ring cell carcinoma of colorectum - current perspectives and molecular biology. Int J Colorectal Dis 2011;26:127-133.

-6 Chen JS, Hsieh PS, Chiang JM, et al: Clinical outcome of signet ring cell carcinoma and mucinous adenocarcinoma of the colon. Chang Gung Med J 2010;33:51-57.

7 Kelemen LE, Koel M: Mucinous carcinomas of the ovary and colorectum: different organ, same dilemma. Lancet Oncol 2011;12:1071-1080.

8 Fu KI, Sano Y, Kato S, et al: Primary signet-ring cell carcinoma of the colon at early stage: a case report and a review of the literature. World J Gastroenterol 2006;12:3446-3449.

$>9$ Hugen N, Verhoeven RH, Lemmens VE, et al: Colorectal signet-ring cell carcinoma: benefit from adjuvant chemotherapy but a poor prognostic factor. Int J Cancer 2015;136:333-339.

-10 Tung SY, Wu CS, Chen PC: Primary signet-ring cell carcinoma of colorectum: an age- and sex-matched controlled study. Am J Gastroenterol 1996;91:2195-2199.

11 Kang H, O'Connell JB, Maggard MA, et al: A 10-year outcomes evaluation of mucinous and signet-ring cell carcinoma of the colon and rectum. Dis Colon Rectum 2005;48:1161-1168.

-12 Kang SH, Chung WS, Hyun CL, et al: A rare case of a signet ring cell carcinoma of the colon mimicking a juvenile polyp. Gut and Liver 2012;6:129-131

13 Sung CO, Seo JW, Kim KM, et al: Clinical significance of signet-ring cells in colorectal mucinous adenocarcinoma. Mod Pathol 2008;21:1533-1541.

14 Sim HL, Tan KY, Poon PL, et al: Primary rectal signet ring cell carcinoma with peritoneal dissemination and gastric secondaries. World J Gastroenterol 2008;14:2118-2120.

-15 Ogino S, Brahmandam M, Cantor M, et al: Distinct molecular features of colorectal carcinoma with signet ring cell component and colorectal carcinoma with mucinous component. Mod Pathol 2006;19:59-68.

16 Darwanto A, Kitazawa R, Maeda S, et al: MeCP2 and promotor methylation cooperatively regulate Ecadherin gene expression in colorectal carcinoma. Cancer Sci 2003;94:442-447.

17 Sargent DJ, Shi Q, Yothers G, et al: Prognostic impact of deficient mismatch repair in 7,803 stage II/III colon cancer patients: a pooled individual patient data analysis of 17 adjuvant trials in the ACCENT database. J Clin Oncol 2014;32(suppl 5):abstr 3507.

18 Secco GB, Fardelli R, Campora E, et al: Primary mucinous adenocarcinoma and signet-ring cell carcinomas of colon and rectum. Oncology 1994;51:30-34. 
Park et al.: Signet-Ring Cell Carcinoma of the Colon: A Case Report and Review of the Literature
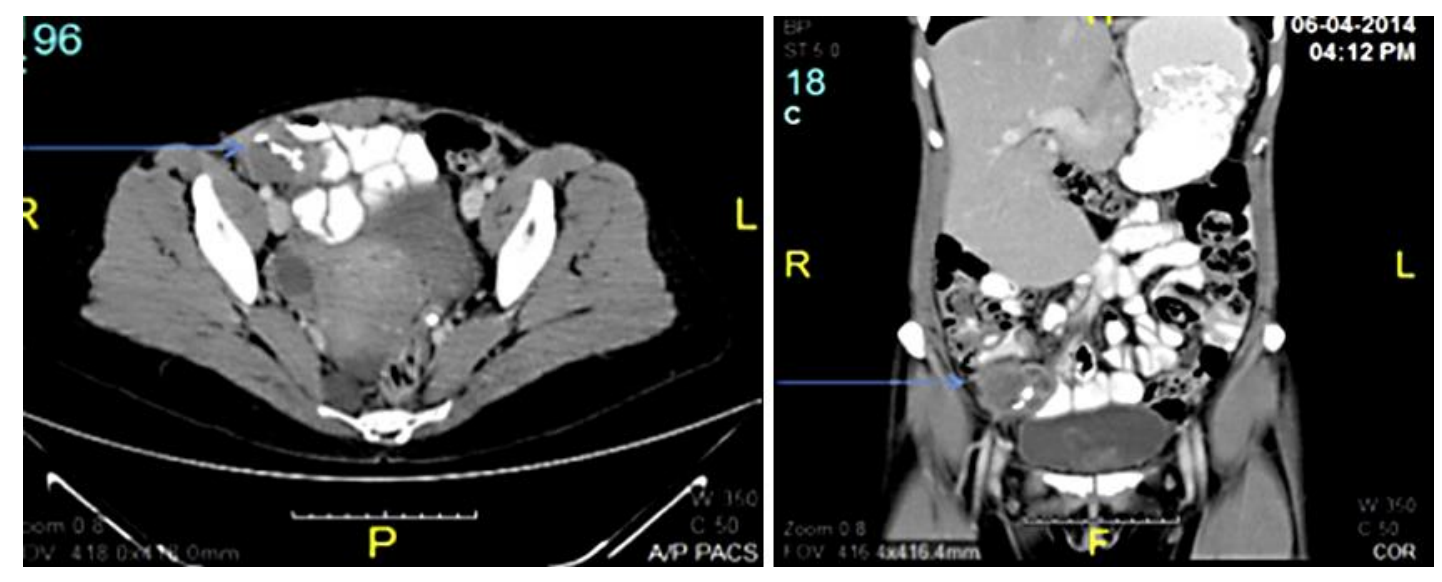

Fig. 1. CT of the abdomen and pelvis with contrast of the patient. There is a lobulated septated cystic mass originating from the cecum (blue arrow). There is an enhancement of the mass's walls as well as focal areas of coarse calcification measuring $4.9 \times 3.5 \times 3.1 \mathrm{~cm}$. A small amount of fluid inferior to this mass is also noted.

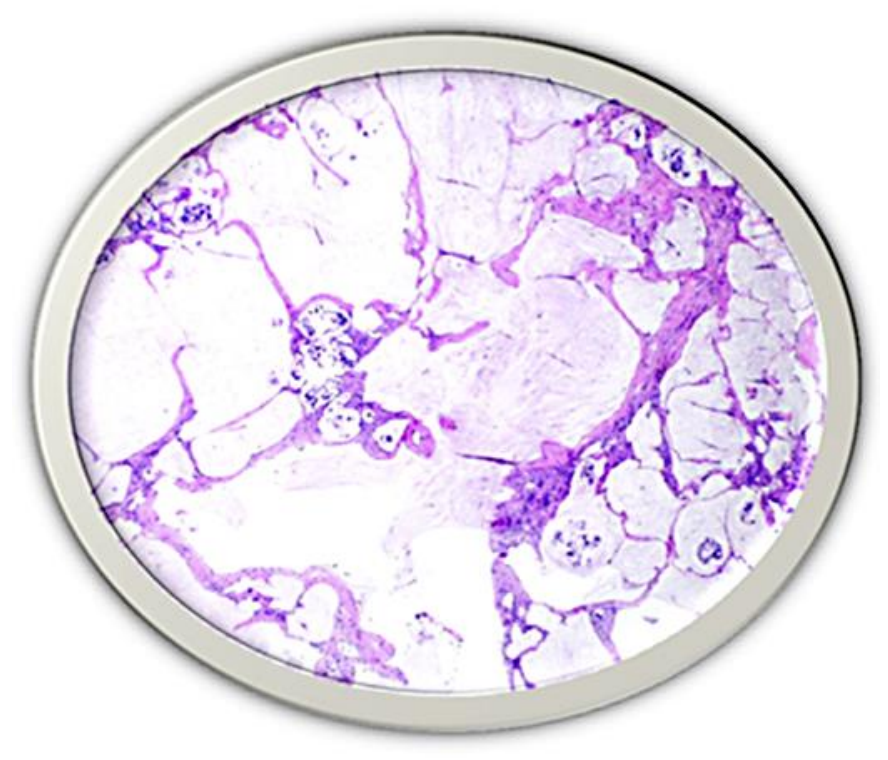

Fig. 2. The biopsy specimen reveals large pools of extravasated mucin invading deep into pericolonic adipose tissue, with variably sized clusters and glandular arrangements of floating malignant cells. HE stain. $\times 40$. 
Park et al.: Signet-Ring Cell Carcinoma of the Colon: A Case Report and Review of the Literature

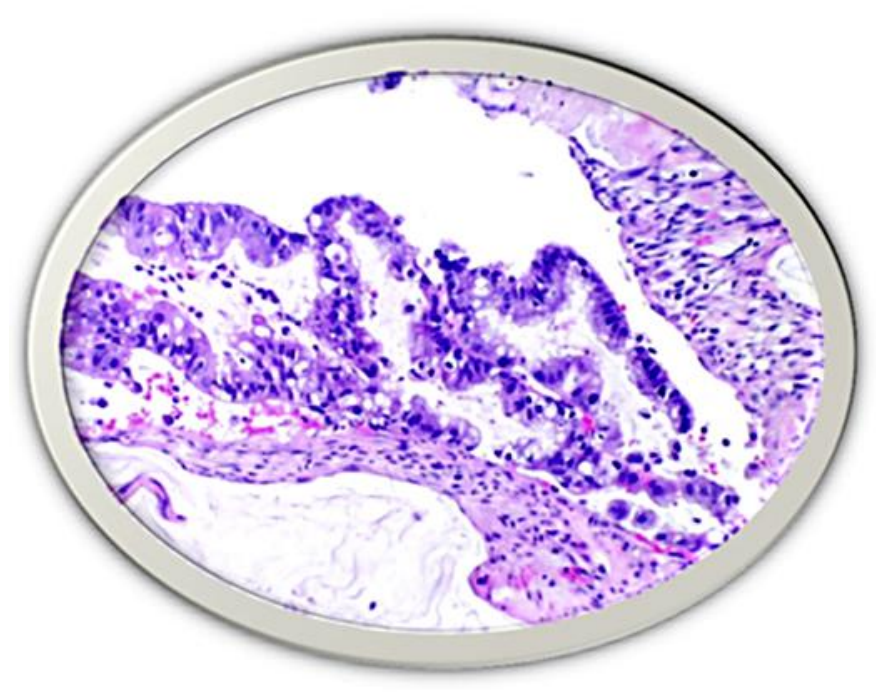

Fig. 3. Focal classical columnar strips of malignant moderately differentiated AC are present. HE stain. $\times 400$.

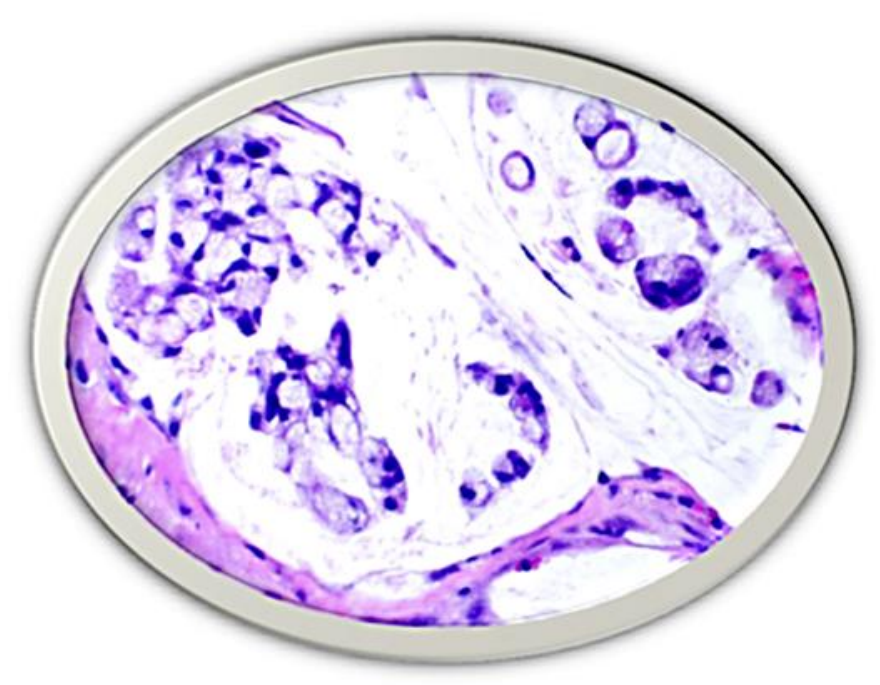

Fig. 4. The majority of the cellularity is composed of clusters and single malignant poorly differentiated signet-ring cells floating within abundant extracellular mucin pools. HE stain. $\times 600$. 